\title{
The effect of phase-reversed and flashed presentations upon the apparent contrast of sinusoidal gratings
}

\author{
FREDERICK L. KITTERLE \\ University of Toledo, Toledo, Ohio
}

\begin{abstract}
The effect of duration upon the apparent contrast of a $0.7 \cdot$ and $7.0-\mathrm{c} / \mathrm{deg}$ sinusoidal grating was determined for two modes of temporal presentation: phase reversals and on-off flashes. For both spatial frequencies at durations of less than $60 \mathrm{msec}$, the apparent contrast of phase-reversed presentations was less than that of on-off flashes. However, for the 0.7-c/deg target at durations of 100-300 msec, the apparent contrast of the phase-reversed presentations was significantly greater than that of on-off presentations. There was no difference in the effect of the two modes of temporal presentation upon apparent contrast at longer durations with the 7.0-c/deg grating. These results are discussed in terms of the role of response transients in the perceived contrast of suprathreshold gratings.
\end{abstract}

Rapid temporal changes in luminance appear to be accentuated by the visual system. For example, electrophysiological data show that the heightened neural activity that accompanies the onset of a target rapidly declines over the duration of stimulus presentation. Similar bursts of neural activity are found at target offset. The inference that a similar temporal pattern of neural activity exists in the human visual system has been derived from psychophysical studies of early light and dark adaptation. It has been found that the detectability of a target is most impaired when the target is presented at the same time that an adapting field is either turned on or off.

Several mathematical models have been developed to account for neural response transients. Although the details of these models may differ, there is a general consensus that transient activity is the result of a visual system whose response is biphasic; that is, consists of an early excitatory component and a delayed inhibitory response. Thus, the lower level of neural activity following the onset of a target is assumed to reflect the influence of delayed inhibition.

While there may be agreement about the role of delayed inhibition in the generation of transient activity and about the stimulus conditions necessary to produce response transients, there is considerably less agreement about their role in the Broca-Sulzer effect, or "temporal brightness enhancement" (Broca $\&$ Sulzer, 1902). This refers to the finding that the brightness of intermediate-duration flashes is greater than that of long-duration flashes.

Several theories have attempted to explain this phenomenon in terms of transient activity. McDougall (1904) suggested that a longer flash whose sensation

The author's mailing address is: Department of Psychology, University of Toledo, Toledo, $\mathrm{OH} 43606$. rises to a maximum and then drops off very sharply should appear dimmer when compared with a briefer flash of the same luminance. The reason for this is that the total sensation (the average of the initial sharp sensation and the final decline of that sensation) is lower than that evoked by a briefer flash which yields only the sharp sensation with little decline in that sensation.

Adrian (1928) showed that the neural response of the visual system of the conger eel, elaborated over time, initially shows a high rate of neural activity at the onset of a visual stimulus, which decays to a lower rate of activity with prolonged presentation. Because this function is similar in shape to that describing the relationship between brightness and duration, Adrian concluded that the changes in brightness with duration reflected an increase and subsequent decrease in neural activity.

A modified version of the transient theory, developed by Baumgardt and Segal (1947), has led to a whole class of modern theories that describe transient changes in visual response in terms of adaptation via negative feedback. The Broca-Sulzer effect is considered to reflect the properties of a mechanism in which an excessive response is eventually adjusted to a proper level for that stimulation as a result of delayed inhibition.

One problem with this analysis is that physiological data represent neural activity as a function of time generated by a flash of a given duration, whereas psychophysical data reflect changes in brightness as a function of duration (Wasserman \& Kong, 1974). In order to relate the two domains, assumptions must be made about how neural responses elaborated over time are related to the brightness of flashes that differ in duration. If brightness is correlated with the initial transient, the magnitude of the transient should 
change in a nonmonotonic fashion with duration. However, Wasserman and Kong (1974) showed that the initial transient measured electrophysiologically in the limulus, changed monotonically with duration. They concluded that the relationship between temporal brightness enhancement and transient neural activity was "illusory"; transients do not play a role in temporal brightness enhancement. This conclusion may need to be reevaluated in light of recent research.

Further insights into the role of response transients in temporal brightness enhancement have been provided by studies that have determined the effect upon temporal brightness enhancement of altering the magnitude of the response transients. This was done by manipulating the temporal waveform of the target (i.e., its rise and/or fall time). It has been shown that when a target is turned on or off gradually, the magnitude of the associated transient activity is reduced (Matsumura, 1976). Kitterle and Corwin (1983) found that temporal contrast enhancement obtained with low-spatial-frequency gratings (Kitterle \& Corwin, 1979) was completely abolished by ramping on a stimulus. A related phenomenon, temporal darkness enhancement (the enhanced perception of the darkness of a luminance decrement exposed for 80 $150 \mathrm{msec}$ ), was also abolished if the onset of the luminance decrement was gradual (Kitterle \& Toney, 1983). Transient activity is abolished by flicker adaptation (Breitmeyer, Levi, \& Harwerth, 1981). Kitterle and Beard (1983) have shown that temporal contrast enhancement is not found under flicker adaptation. This result provides additional evidence for the role of transients in sensory coding. The present study compares the effects of phase reversals and on-off flashes on apparent contrast. Differences between these modes of presentation should provide further evidence for the role of transients and delayed inhibition in temporal enhancement effects.

A comparison of these two forms of temporal modulation at threshold indicates that a low spatial frequency grating that is phase shifted (i.e., the light and the dark bars are repetitively exchanged over time) produces a measure of sensitivity twice that obtained with on-off stimulation (i.e., when the grating is simply turned on and off repetitively) (Kulikowski \& Tolhurst, 1973). A similar result is obtained when sensitivity for detecting large bars presented as luminance reversals and sensitivity for detecting onoff flashes are compared (Stromeyer, Zeevi, \& Klein, 1979). Luminance and phase reversals may have elevated sensitivity because they are optimal modes of stimulation for a mechanism that has a biphasic impulse response (Sperling \& Sondhi, 1968; Watson \& Nachmias, 1977; Kelly, 1971a, 1971b). Thus, apparent contrast might be greater for low-spatialfrequency gratings presented as phase reversals than for those flashed on and off. With high spatial frequency gratings, there is no difference in sensitivity between these two modes of temporal presentation. The impulse response for this stimulus does not show any delayed inhibitory component (Kelly, 1971b). Transients do not appear to accompany either the onset or the offset of high-spatial-frequency targets (Mitov, Vassilev, \& Manahilov, 1981). In addition, temporal contrast enhancement is not found with high spatial frequency gratings (Kitterle \& Corwin, 1979). This suggests that there should be no difference between the two modes of stimulation for high spatial frequency gratings. The present experiment tests these predictions.

\section{METHOD}

\section{Observers}

Two observers participated in this experiment; K.K. (22 years) and the author, F.L.K. (40 years). Both had normal visual acuity and were highly practiced psychophysical observers. K.K. was naive about the hypothesis of this experiment.

\section{Apparatus}

The stimuli were sinusoidal gratings presented by means of a two-channel haploscopic system. Gratings with two spatial frequencies were used: 0.7 and $7 \mathrm{c} / \mathrm{deg}$. Each channel consisted of an oscilloscope (Tektronix 5103/DN, P-31 phosphor) upon which the gratings were generated using conventional methods. Both oscilloscopes were masked down to circular apertures that at a viewing distance of $167 \mathrm{~cm}$ subtended a visual angle of $4.5 \mathrm{deg}$. The screens were spatially adjacent and separated by 2 deg. Midway between them was a dim red fixation light. The mean luminance of the screens was $9.5 \mathrm{~cd} / \mathrm{m}^{2}$. During the course of the experimental session, care was taken to ensure that the mean luminance of both screens matched. The contrast of the gratings was $27 \%$. All luminance calibrations were made by means of an EG\&G photometer/radiometer. A Sym 1 microprocessor (Synertek Corp.) was used to control the timing of the stimulus presentations and the collection of data. A browrest and head holder provided for steady viewing.

\section{Procedure}

After a 5-min adaptation to the mean luminance of the display, the experiment began. On a given trial, the task of the observer was to determine which of two briefly flashed sinusoidal gratings of the same spatial frequency had the greater apparent contrast. The observer indicated his response by pressing one of two microswitches. Both of the targets had the same physical contrast, but on a given trial one of the targets was presented for a duration of $\mathrm{T}$ msec (the on-off condition). The other target was presented for a duration $T / 2 \mathrm{msec}$ in one phase and then for an additional $\mathrm{T} / 2 \mathrm{msec} 180 \mathrm{deg}$ out of phase. There was a $1-\mathrm{sec}$ intertrial interval. Flash duration as well as the channel in which the on-off or contrast reversal stimulus was presented varied randomly over trials. Within an experimental session, apparent contrast judgments were made for one spatial frequency. Twenty replications were made at each exposure duration. The entire experiment was repeated four times.

\section{RESULTS}

The data are presented in a format similar to that developed by Bowen and Pokorny (1978). The percent of trials on which the apparent contrast of the grating presented in the reversal mode was judged to have a greater contrast than the on-off grating is plotted as a function of the duration of the on-off 
grating. The results for Observer K.K. are shown in Figure 1A, and the results of Observer F.L.K. are presented in Figure 1B. The data for the $0.7-\mathrm{c} / \mathrm{deg}$ grating are shown as open circles, and the data for the 7-c/deg target are plotted by filled circles. Note that there is fairly good agreement between observers.

A binomial test based upon average data was used to determine the range of durations over which the data deviated significantly from $50 \%$. For the $0.7-$ $\mathrm{c} / \mathrm{deg}$ target (open circles), the phase-reversed grating was judged significantly greater than the on-off stimuli for flash durations in the range of $100-360 \mathrm{msec}$. There was no evidence in data obtained with the 7.0$\mathrm{c} / \mathrm{deg}$ grating (filled circles) that the phase-reversal condition was ever judged to be significantly greater than the on-off presentation. Below $60 \mathrm{msec}$, the onoff presentations yielded greater perceived contrast

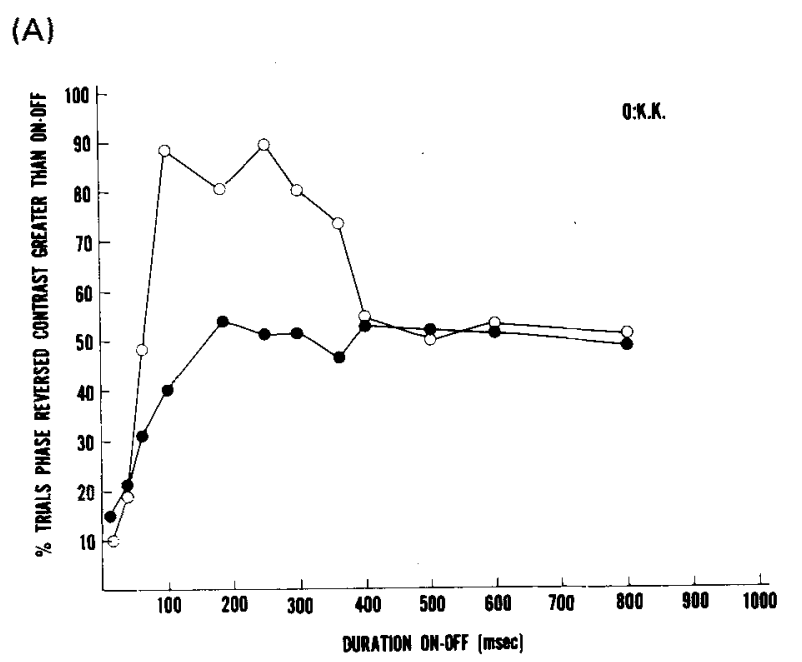

(B)

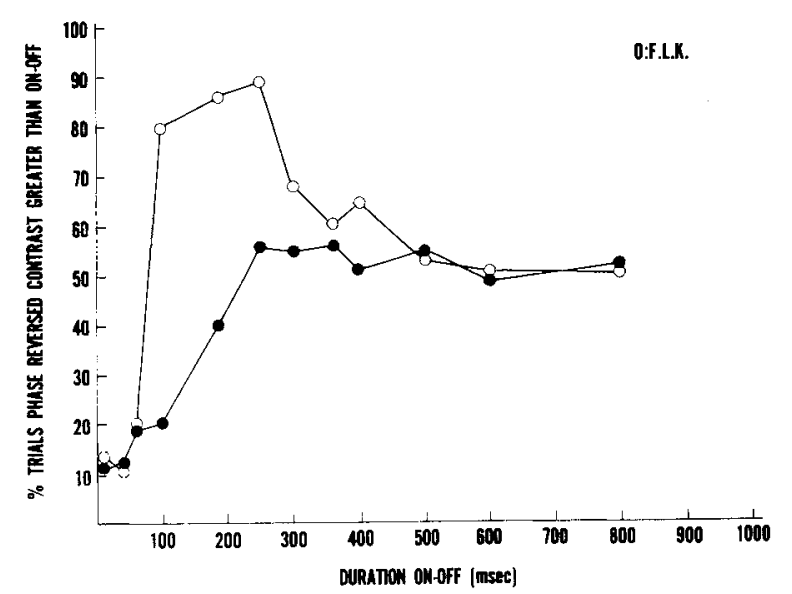

Figure 1. The percentage of trials on which a phase-reversed grating was judged to have greater contrast than an on-off flash as a function of the duration of the on-off flash. The open circles plot the data for the 0.7-c/deg gratings and the filled circles, the data for the 7.0-c/deg grating. (A) Results for Observer K.K. (B) Results for Observer F.L.K. than the phase reversal conditions for both the 0.7and $7.0-\mathrm{c} / \mathrm{deg}$ targets. Both figures show that the curve describing the results for the $7.0-\mathrm{c} / \mathrm{deg}$ grating rises more slowly than that obtained with the 0.7 c/deg target and that it asymptotes at $200-300 \mathrm{msec}$.

In summary, the data obtained with the $0.7-\mathrm{c} / \mathrm{deg}$ grating indicate that, for durations less than $60 \mathrm{msec}$, the apparent contrast of gratings presented in the onoff mode is greater than that of gratings presented as phase reversals. However, this reverses with durations in the range of 100-300 msec. The apparent contrast is greater for phase-reversed gratings than for gratings presented in the on-off mode. Beyond $400 \mathrm{msec}$, there is little difference between the two modes of temporal presentation. This pattern of results is not found with the 7-c/deg grating. With this target, at durations less than $60 \mathrm{msec}$ the apparent contrast of gratings presented in the on-off condition is greater than it is with phase-reversed targets. At longer durations, there is virtually no difference in the effects of the two modes of presentation upon apparent contrast.

\section{DISCUSSION}

Previous experiments on temporal contrast enhancement have compared the apparent contrast of flashes that vary in duration against a standard that is usually a steady stimulus or one presented for a relatively long duration (e.g., Kitterle \& Corwin, 1979). The present study differed from those earlier experiments in that observers judged the apparent contrast of two flashes that were presented for the same duration One was flashed on and off (on-off mode), and the other consisted of a grating that was presented in one phase for half the presentation time and in another phase for the other half (i.e., the bright bars of the grating were exchanged for dark bars, and the dark ones were exchanged for light ones-luminance reversal mode). The results of this experiment indicate that, for low-spatial-frequency gratings at durations in which temporal contrast enhancement is found (i.e., $70-120 \mathrm{msec}$ ), the apparent contrast is greater for luminance reversals than for on-off flashes.

In previous work with on-off presentations, Kitterle and Rysberg, (1976) and Kitterle and Corwin (1979) have shown that the apparent contrast of low (not high) spatial frequency sinusoidal gratings is greater at intermediate durations than at longer or shorter presentations. Since response transients accompany the onset and offset of low spatial frequency gratings, we have concluded that transients are necessary for obtaining temporal contrast enhancement. Our later work supports this conclusion by showing that conditions that eliminate response transients abolish temporal contrast enhancement (Kitterle \& Corwin, 1983; Kitterle \& Beard, 1983; Kitterle \& Toney, 1983). 
The fact that luminance reversals produce a greater temporal contrast enhancement effect than do on-off presentations argues strongly for the role of response transients in temporal contrast enhancement.

In the spatiotemporal frequency domain, contrast sensitivity is a bandpass function of temporal frequency at low spatial frequencies and a low-pass function at high spatial frequencies, and vice versa (Robson, 1966). From elementary linear analysis, it follows that the temporal response of the visual system to low-and high-spatial-frequency gratings should differ (Kelly \& Savoie, 1978; Kulikowski \& Tolhurst, 1973).

Consider the response to a stimulus that is switched on suddenly and then left on for an extended period of time. With a temporal bandpass function with a pronounced attenuation at low temporal frequencies, the response will be transient, returning to a resting level rapidly. When the stimulus is removed, the response will be a transient of opposite sign. If, on the other hand, the temporal response is low-pass, then the response will be at some maintained level until the pattern is extinguished. In essence, response transients will not accompany either flash onset or flash offset. Several studies have empirically verified this at threshold levels (e.g., Tolhurst, 1975) and also at suprathreshold levels (Green, 1981; Kitterle, Corwin, \& Berta, 1979; Mitov et al., 1981).

When a pattern is presented as a luminance reversal, a low-pass mechanism will produce a response that will vary a fixed amount above and below the resting level. Both on-off and luminance reversal presentations will produce similar peak responses, although the depth of modulation for the alternated pattern will be twice that of the on-off presentation. If the apparent contrast of high-spatial-frequency gratings depends upon the magnitude of the peak response, both modes of stimulation should yield the same apparent contrast. This appears to be the case. As can be seen in Figures 1A and 1B, at long durations there is virtually no difference in apparent contrast between the two modes of temporal presentation. Below $300 \mathrm{msec}$, the apparent contrast of luminance-reversal presentations is less than on-off flashes. This is probably due to the partial cancellation caused by temporal summation of responses of opposite polarity (Ikeda, 1965). A similar reduction, but a shorter duration, is found with low-spatial-frequency gratings and reflects differences in the limits of temporal summation of contrast (see Kitterle \& Corwin, 1979; Legge, 1978).

A temporal bandpass filter, as mentioned earlier, yields transients at onset and offset; its response to on-off and luminance reversals differs. The response to an on-off presentation varies between the peak of the on response and the minimum of the off response. However, with a luminance reversal, both the amplitude of the initial transient and the depth of modulation are doubled. Consequently, if the initial transient plays a role in the TCE, luminance reversals should yield a greater apparent contrast than on-off flashes. Clearly, this is the case, as can be seen at intermediate durations for low-spatialfrequency gratings in Figures 1A and 1B. However, beyond $500 \mathrm{msec}$, the mode of temporal presentation does not appear to have any effect. This can be accounted for in terms of the temporal frequency content of the flash. At longer durations, there are fewer high temporal frequency Fourier components than at shorter durations.

Low-pass and bandpass temporal functions are thought to reflect characteristics of different mechanisms (e.g., Kelly, 1971a, 1971b; Kelly \& Savoie, 1978). Impulse response functions have been derived for these two temporal functions. The impulse response for bandpass functions is biphasic, consisting of an early positive component and a delayed negative component. The impulse response for the lowpass function does not have any negative component. One view has been that these functions may reflect the activity of a single channel such as a receptive field with spatially antagonistic regions-center excitatory and surrounding inhibitory regions. The monophasic response is thought to reflect the temporal response of the center region, whereas the biphasic impulse response function has been thought to reflect the temporal response of an early excitatory response and a delayed inhibitory response from the surround regions. Impulse response functions have been derived for these two temporal functions. The impulse response for bandpass functions is biphasic, consisting of an early positive component and a delayed negative component. The impulse response for the low-pass function does not have any negative component.

There has been a long history of suggestions that temporal brightness enhancement reflects the properties of an underlying mechanism with a biphasic temporal response (e.g., Adrian, 1928; Baumgardt \& Segal, 1947). These earlier studies have led to more sophisticated mathematical models to account for transient-type responses (e.g., Kelly, 1971a). The results of the present study provide empirical evidence for the necessary connection between transient responses and temporal enhancement. Other studies suggest, however, that although transients are necessary for obtaining temporal enhancement effects, they may not be sufficient (Bowen, Sekuler, Owsley, \& Markell, 1982). Although response transients may accompany brief flash presentations, criterion effects may influence how observers utilize this sensory information in judging brightness or contrast.

\section{REFERENCES}

Adrian, E. D. (1928). The basis of sensation: The action of sense organs. London: Christophors. 
Baumgardt, E., \& Segal, J. (1947). Facilitation et inhibition, parametres de la fonction visuelle. Annee Psychologie, 43-44, 54-102.

Bowen, R. W., \& Pokonny, J. (1978). Target edge sharpness and temporal brightness enhancement. Vision Research, 18, 1691-1695.

Bowen, R. W., Sekuler, R., Owsley, C. J., \& Markell, K. A. (1982). Individual differences in pulse brightness perception. Perception \& Psychophysics, 30, 587-593.

Breitmeyer, B., Levi, D. M., \& Harwerth, R. S. (1981). Flicker masking in spatial vision. Vision Research, 21, 1387-1394.

Broca, D., \& Sulze R, A. (1902). La sensatin luminise en fonction du temps. Journal de Physiologie, 4, 632-640.

Green, M. A. (1981). Psychophysical relationships among mechanisms sensitive to pattern, motion and flicker. Vision Research, 21, 971-984.

IKEDA, M. (1965). Temporal summation of positive and negative flashes in the visual system. Journal of the Optical Society of America, 55, 1527-1534.

KELLY, D. H. (1971a). Theory of flicker and transient responses. 1. Uniform fields. Journal of the Optical Society of America, 61, 537-546.

KeLLY, D. H. (1971b). Theory of flicker and transient responses. II. Counterphase gratings. Journal of the Optical Society of America, 61, 532-640.

Kelly, D. H., \& Savoie, R. E. (1978). Theory of flicker and transient responses. III. An essential nonlinearity. Journal of the Optical Society of America, 68, 1481-1490.

KitTerle, F. L., \& BeARD, B. L. (1983). The effects of flicker adaptation upon temporal contrast enhancement. Perception \& Psychophysics, 33, 75-78.

KitTE RLE, F. L., \& CoRwin, T. R. (1979). Enhancement of apparent contrast in flashed sinusoidal gratings. Vision Research, 19, 33-39.

KitTerle, F. L., \& Corwin, T. R. (1983). The effects of temporal waveform upon apparent contrast. Perception \& Psychophysics, 33, 72-74.

Kitterle, F. L., Corwin, T. R., \& Berta, J. (1979). Masking of sinusoidal targets by uniform fields of unequal duration. Journal of the Optical Society of America, 69, 1445.

KitTenle, F. L., \& Rysberg, J. A. (1976). The effect of dura- tion magnitude estimates of apparent contrast in sine-wave gratings. Perception \& Psychophysics, 19, 335-338.

KitTe RLE, F. L., \& Toney, H. C. (1983). The effects of temporal waveform upon temporal darkness enhancement. Perception \& Psychophysics, 33, 375-378.

Kulikowski, J. J., \& Tolmurst, D. J. (1973). Psychophysical evidence of sustained and transient detectors in human vision. Journal of Physiology (London), 232, 149-162.

LEGGE, G. E. (1978). Sustained and transient mechanisms in human vision: Temporal and spatial properties. Vision Research, $18,69-81$.

Matsumura, M. (1976). Visual masking by luminance increment and decrement: Effects of rise time and decay time. Tohoku Psychologia Folia, 35, 104-114.

McDougall, W. (1904). The variation of the intensity of visual sensation with the duration of the stimulus. British Journal of Psychology, 1, 151-189.

Mitov, D., Vassilev, A., \& Manahilov, V. (1981). Transient and sustained masking. Perception \& Psychophysics, 30, 205210.

RoBson, J. G. (1966). Spatial and temporal contrast sensitivity functions of the human eye. Journal of the Optical Society of America, 56, 1141.

Sperling, G., \& Sondhi, M. M. (1968). Model for luminance discrimination and flicker detection. Journal of the Optical Society of America, 58, 1133-1145.

Stromeyer, C. F., ZeeVi, Y. Y., \& KLein, S. (1979). Response of visual mechanisms to stimulus onsets and of fsets. Journal of the Optical Society of America, 69, 1350-1354.

Tolnurst, D. J. (1975). Sustained and transient channels in human vision. Vision Research, 15, 1151-1155.

Wasserman, G. S., \& Kong, K. L. (1974). Illusory correlation of brightness enhancement and transients in the nervous system. Science, 184, 911-913.

Watson, A. B., \& Nachmias, J. (1977). Patterns of temporal interaction in the detection of gratings. Vision Research, 17, 893-902.

(Manuscript received September 23, 1983; revision accepted for publication May 21, 1984.) 